International Journal of Biomedicine I June 2019 - Volume 9, Issue Suppl_1: Abstracts From the Second Russian International Conference "Cryo-electron microscopy 2019: achievements and prospects"

POSTER ABSTRACT PRESENTATIONS

SESSION TITLE: APPLICATIONS OF CRYO-EM IN MEDICINE

DOI: 10.21103/IJBM.9.Suppl_1.P36

\title{
Abstract P-36: HBc4M2e Virus-Like Particles for Influenza A Vaccine Development: a Cryo-EM Study
}

\author{
Alexey V. Shvetsov ${ }^{1,2}$, Evgeny B. Pichkur ${ }^{1,3,5}$, Tatiana A. Shtam ${ }^{1}$, Vladimir V. Egorov ${ }^{1,4}$, \\ Liudmila M. Tsybalova ${ }^{4}$, Liudmila A. Stepanova ${ }^{4}$, Aram A. Shaldzhyan ${ }^{1,4}$, Yana A. Zabrodskaya ${ }^{1,2,4}$ \\ ${ }^{1}$ NRC «Kurchatov Institute» - PNPI, Gatchina, Russia; ${ }^{2}$ Peter the Great St. Petersburg Polytechnic \\ University, Saint-Petersburg, Russia; ${ }^{3}$ NRC «Kurchatov Institute», Moscow, Russia; ${ }^{4}$ Smorodintsev \\ Research Institute of Influenza, Saint-Petersburg, Russia; ${ }^{5}$ FSRC “Crystallography and Photonics", \\ Moscow, Russia
}

Background: Currently, various virus-like particles (VLPs) are widely used in antiviral vaccines design, including Hepatitis B virus core antigen (HBc)-based VLPs (Blokhina et al. 2013 doi:10.1016/j.virol.2012.09.014). In this work, a structure of HBc-based VLP with the antigenic insert corresponding to the highly conserved region of the M2E protein antigen of the influenza (fourfold repetition) was characterized. This study is essential for optimization of antigenic insertion sequence and increasing the effectiveness of an existing universal flu vaccine.

Methods: Cryo-EM data was collected using cryo-TEM Krios (Thermo-Fisher, USA) at 300kV using DED Falcon II. Dataset was processed using Relion (Scheres et al. 2012 doi:10.1016/j.jsb.2012.09.006, Scheres et al. 2012 doi:10.1016/j.jmb.2011.11.010) and CisTEM (Grant et al. 2018 doi:10.7554/elife.35383). Refinement of the full-atom structure of electron density maps was carried out using the Phenix (Adams et al. 2010 doi:10.1107/S0907444909052925, Grant et al. 2018 doi:10.7554/elife.35383) and Gromacs (Abraham et al. 2015 doi:10.1016/j.softx.2015.06.001) software packages using the Gromacs densfit module (Igaev et al. 2019 doi:10.7554/elife.43542).

Results: It was found that HBc4M2E VLPs have icosahedral symmetry and, like HBc VLPs, can form structures with symmetry $\mathrm{T}=3$ and $\mathrm{T}=4$ (180 monomers and 240 monomers, respectively). It was noteworthy that, in contrast to HBc VLPs in which particles with $\mathrm{T}=4$ symmetry predominate (about $80 \%$ ), particles with $\mathrm{T}=3$ symmetry (about $70 \%$ ) predominate in HBc4M2E VLPs. In this case a fragment of $\mathrm{M} 2 \mathrm{E}$ repeats situated in the $\mathrm{HBc}$ antigenic loop are located on the outer surface of the VLP.

Conclusion: Thus, as a result of CryoEM-based study, the structures peculiar to virus-like particles carrying the antigen of influenza A virus were characterized. Results obtained allow us to optimize number of $\mathrm{M} 2 \mathrm{E}$ repetitions and primary structure of $\mathrm{HBc}$ antigenic insert for further universal flu vaccine development. 
Key Words: vaccine design • structure $\bullet$ cryo-EM • virus-like particles

Sources of Funding: This work was supported by grant \#19-74-20146 by RSCF.

The authors acknowledge the support and the use of resources of the Resource Center for Probe and Electron Microscopy at the NRC "Kurchatov Institute. The authors acknowledge the support and the use of computing resources of the Federal Collective Usage Center "Complex for Simulation and Data Processing for Mega-Science Facilities"

International Journal of Biomedicine. 2019;9 Suppl 1: S32-33. doi: 10.21103/IJBM.9.Suppl_1.P36

(C)2019 International Medical Research and Development Corporation 